\title{
Estudio de la Catálisis Heterogénea con Disulfuro de Hierro (II) como Tratamiento de Emisiones Gaseosas Contaminadas con Tolueno Producidas en la Industria de Pintura
}

\author{
Cabrera, Marcelo $^{1, *}$ (iD) Montenegro, Lucía $^{2}$ iD $;$ Mejía Stephanie $^{2}$ iD \\ ${ }^{1}$ Universidad Internacional del Ecuador, Escuela de Gestión Ambiental, Quito, Ecuador \\ ${ }^{2}$ Escuela Politécnica Nacional, Facultad de Ingeniería Química y Agroindustria, Quito, Ecuador
}

\begin{abstract}
Resumen: Esta investigación pretende desarrollar una alternativa para el tratamiento de las emisiones gaseosas contaminadas con tolueno producidas en la industria de pinturas, de modo que se minimice el riesgo de afectación a la salud de trabajadores y personas aledañas que mantienen contacto con dichas emisiones. Para simular el caudal del aire contaminado, se desarrolló a nivel de laboratorio un prototipo de un sistema fundamentado en el proceso de evaporación, a través del cual se obtuvo una concentración de tolueno igual a 108 partes por millón (ppm) en la corriente de aire. Consecutivamente, se diseñó y desarrolló un sistema para tratar la corriente, compuesto por el agente oxidante peróxido de hidrógeno $\left(\mathrm{H}_{2} \mathrm{O}_{2}\right)$ y el catalizador disulfuro de hierro $\mathrm{II}\left(\mathrm{FeS}_{2}\right)$. En una primera etapa, se estudió la eficacia del $\mathrm{H}_{2} \mathrm{O}_{2}$ como agente de tratamiento obteniéndose que a un flujo de $2 \mathrm{~mL} / \mathrm{min}$, se degrada el 10,1\% del tolueno presente en la emisión contaminada, mientras que a un flujo de $3 \mathrm{~mL} / \mathrm{min}$, se degrada el 10,5\%. A continuación, se trabajó con diferentes cantidades de disulfuro de hierro II en una concentración igual al $86 \%$. Se determinó que $10 \mathrm{~g}$ de $\mathrm{FeS}_{2}$ degradan al tolueno en un 36,25\%, mientras que 20 gramos un 61,09\% y $30 \mathrm{~g}$ un 70,39 $\%$. Finalmente, se concluyó que la concentración de tolueno en el aire disminuye hasta $32 \mathrm{ppm}$, cuando se trabaja bajo las mejores condiciones determinadas de $2 \mathrm{~mL} / \mathrm{min}$ de $\mathrm{H}_{2} \mathrm{O}_{2}$ y $30 \mathrm{~g}$ de $\mathrm{FeS}_{2}$.
\end{abstract}

Palabras clave: Tolueno, catálisis heterogénea, emisiones gaseosas, oxidación.

\section{Study of Heterogeneous Catalysis with Iron (II) Disulfide as a Treatment in Gaseous Emissions Contaminated with Toluene from Paint Industries}

\begin{abstract}
This research aims to develop an alternative for the treatment of gaseous emissions contaminated with toluene from paint industries, so that it minimizes the risk of affecting the health of workers and neighbors who come into contact with such emissions. To simulate the flow of contaminated air, a prototype of a system based on the evaporation process was developed at laboratory, through which a concentration of toluene equal to 108 parts per million (ppm) was obtained in the air stream. Consequently, a system was designed and developed to treat the stream, composed of the oxidizing agent hydrogen peroxide $\left(\mathrm{H}_{2} \mathrm{O}_{2}\right)$ and the catalyst iron disulfide II $(\mathrm{FeS})_{2}$. First, the effectiveness of $\mathrm{H}_{2} \mathrm{O}_{2}$ as a treatment agent was studied, obtaining that at a flow rate of $2 \mathrm{~mL} / \mathrm{min}$ degraded a $10.1 \%$ of the toluene present in the contaminated emission, while a flow rate of $3 \mathrm{~mL} / \mathrm{min}$ degraded a $10.5 \%$. Next, the effect of the addition of different amounts of iron II disulfide at a concentration equals to $86 \%$, the degradation of toluene was analyzed. It was determined that $10 \mathrm{~g}$ of $\mathrm{FeS}_{2}$ degrade toluene by $36.25 \%$, while $20 \mathrm{~g}$ degrades it by $61.09 \%$ and $30 \mathrm{~g}$ degrades it by $70.39 \%$. Finally, it was concluded that the concentration of toluene in air decreases up to 32 ppm, when working under the best conditions determined of $2 \mathrm{~mL} / \mathrm{min}$ of $\mathrm{H}_{2} \mathrm{O}_{2}$ and $30 \mathrm{~g}$ of $\mathrm{FeS}_{2}$.
\end{abstract}

Keywords: Toluene, heterogeneous catalysis, gaseous emissions, oxidation.

\section{INTRODUCCIÓN}

El tolueno, de fórmula química $\mathrm{C}_{6} \mathrm{H}_{5} \mathrm{CH}_{3}$, es un hidrocarburo líquido de uso frecuente en las industrias como disolvente o como ingrediente en la fabricación de pinturas o pegamentos. El tolueno o metilbenceno forma parte de la lista de compuestos orgánicos volátiles (COVs), cuyos efectos son negativos a nivel medioambiental y en la salud humana (INSHT, 2007, p. 1).

Investigaciones realizadas por el Department of Health and Human Service (HHS) de los Estados Unidos, indican que la 
elaboración de pinturas genera aproximadamente 283000 toneladas de emisiones gaseosas de tolueno por año en concentraciones de entre 99 y 200 partes por millón (ppm), motivo por lo cual esta industria es responsable de aproximadamente el $16 \%$ de las fuentes contaminantes con tolueno. Las personas relacionadas con este tipo de industrias han reportado afecciones crónicas a su sistema nervioso central, fallas en sus riñones y problemas visuales y auditivos (INSHT, 2007, p. 2; DHHS, 2015, p. 302).

En la actualidad, el TULSMA del Ministerio del Ambiente del Ecuador, no regula el valor máximo de concentración de emisiones de los compuestos orgánicos volátiles, no obstante, se ha observado un incremento en la preocupación por controlar este tipo de emisiones. Por esta razón, se realizó un estudio de la calidad del aire atmosférico en la ciudad de Quito, y la vulnerabilidad a los compuestos orgánicos volátiles. En este estudio se concluyó que las personas cercanas a las fuentes de contaminación se ven afectadas por estos aspectos ambientales. Por este hecho, se propuso realizar una investigación que permita minimizar el impacto de los COV's en la salud de las personas (Ministerio del Ambiente del Ecuador, 2015; IFA, 2007, p. 57).

Existen dos tipos de métodos para el tratamiento de COV's, los métodos fisicoquímicos y biológicos. El método fisicoquímico emplea agentes oxidantes para la degradación del tolueno, entre los agentes oxidantes más empleados está el peróxido de hidrógeno $\left(\mathrm{H}_{2} \mathrm{O}_{2}\right)$ el cual actúa junto al hierro como catalizador, para mejorar su eficacia (Cárdenas et al., 2003, p. 30; Castells, 2012, p. 114).

El $\mathrm{FeS}_{2}$ o disulfuro de hierro II es un mineral de gran abundancia en la naturaleza, compuesto aproximadamente por un 46,6 \% de hierro el cual es extraído para su uso en la industria (Klein y Hurbult, 2002 p. 339).

Esta investigación constituye una alternativa factible para el tratamiento de corrientes gaseosas contaminadas con tolueno procedentes de las industrias de pinturas, evitando así la afección a la salud de las personas cercanas a esta industria, así mismo, se podrán usar los resultados de esta investigación para su aplicación en las industrias quiteñas que presenten contaminación en el ambiente con tolueno (Cobo, 2013, p. 134).

\section{MATERIALES Y MÉTODOS}

\subsection{Generación de un flujo constante con tolueno}

\subsubsection{Diseño y construcción del sistema}

Considerando la volatilidad del tolueno, el sistema diseñado y desarrollado a nivel de laboratorio se basó en un proceso de evaporación (EPA, 2000, p.1).

Un caudal controlado de aire ingresó al sistema que contenía tolueno en estado líquido, el cual mantenía una evaporación constante. La mezcla del tolueno evaporado y el aire dieron como resultado una corriente contaminada.
Para mantener un flujo constante de tolueno en la corriente, se controlaron las siguientes variables: temperatura del líquido, temperatura y caudal del aire y, el espacio transversal expuesto del líquido.

Los procedimientos empleados para el control de los parámetros enunciados se presentan a continuación (Montoya, 2002, p. 12):

\section{a. Establecimiento de la temperatura del aire}

A fin de incrementar la temperatura ambiental del flujo de aire desde $16,9^{\circ} \mathrm{C}$ a $20^{\circ} \mathrm{C}$, se implementó y usó un intercambiador de calor. La temperatura indicada inicial del aire es un valor registrado para el 2018, en la ciudad de Quito-Ecuador, por el INAMHI (INAMHI, 2018, p. 14)

Dada la facilidad de construcción y cálculo, el intercambiador tuvo un diseño de tubos concéntricos. Sus dimensiones fueron: espesor de vidrio $1 \mathrm{~mm}$, longitud de $20 \mathrm{~cm}$, diámetro externo del tubo exterior de $1,8 \mathrm{~cm}$ y $1,1 \mathrm{~cm}$ para el tubo interior. A través del área anular de este intercambiador de calor atravesaba agua procedente de un baño María y por los tubos concéntricos, aire (Incrópera et al., 2011, p. 706).

La temperatura del baño termostático requerida para incrementar la temperatura del ambiente hasta los $20^{\circ} \mathrm{C}$, fue calculada a través del modelado de un intercambiador de tubos concéntricos en contracorriente, empleando para ello, la metodología presentada por Kern. El cálculo se realizó para los valores de $10{ }^{\circ} \mathrm{C}$ y $19{ }^{\circ} \mathrm{C}$ como temperaturas ambientales y $0,5 \mathrm{~L} / \mathrm{min}$ y $2,5 \mathrm{~L} / \mathrm{min}$ como datos de caudales de aire. Se consideró que los valores escogidos de temperatura para el cálculo sean mayores a la temperatura mínima reportada por el INAMHI para la ciudad de Quito año 2018 (Kern, 1999; INAMHI, 2018, p. 19).

Con el dato teórico y datos experimentales de los valores de la temperatura del baño termostático requerida para incrementar a $20^{\circ} \mathrm{C}$, la temperatura ambiental se calculó el error existente.

Una vez controlada la temperatura del aire de entrada se desarrollaron mecanismos de control del proceso de evaporación, para ello se analizaron los parámetros del volumen en función del tiempo del aire de ingreso, la temperatura del tolueno líquido y el espacio transversal expuesto.

Los mecanismos empleados se presentan a continuación:

b. Control de la temperatura del tolueno, área de evaporación y flujo del aire

Para controlar el espacio transversal expuesto o área de evaporación, se tomaron dos envases de vidrio de una misma longitud, pero de distinto diámetro y se colocó tolueno dentro de los mismos. La sección transversal de los recipientes utilizados fue de $12,76 \mathrm{~cm}^{2}$ y $2,97 \mathrm{~cm}^{2}$.

Empleando un rotámetro de capacidad $8 \mathrm{~L} / \mathrm{min}$ provisto de válvula reguladora, se controló el caudal de aire de ingreso. Esto fue necesario dado que bibliográficamente se halló que 
existe mayor evaporación a mayor flujo de aire (Pozo, 2012, p. 22).

Los flujos de aire se establecieron a $0,5 \mathrm{~L} / \mathrm{min}$ y $2,5 \mathrm{~L} / \mathrm{min}$. El valor más bajo escogido corresponde a un dato que puede ser medido por el rotámetro en ausencia de aire, mientras que el valor más alto corresponde a un valor cercano de una investigación similar al presente estudio (Bae et al., 2014, p. $32)$.

Con estos criterios se diseñó y desarrolló un medio con una columna de vidrio, cuyas medidas fueron: $6 \mathrm{~cm}$ de diámetro y $55 \mathrm{~cm}$ de alto. En la parte interna de la columna, se colocaron los recipientes de $12,76 \mathrm{~cm}^{2}$ y $2,97 \mathrm{~cm}^{2}$ llenos de tolueno (Wang et al., 2002, p. 63).

Pozo (2012) establece en su análisis de evaporación de tolueno y xileno, que las temperaturas óptimas para el tolueno se encuentran entre los $18{ }^{\circ} \mathrm{C}$ o $25^{\circ} \mathrm{C}$. Conforme a lo descrito, se revistió la columna de vidrio con una chaqueta de calentamiento por la cual circulaba agua proveniente de un baño termostático, alcanzado en la columna las temperaturas mencionadas (p. 43).

Posteriormente, aire a $20{ }^{\circ} \mathrm{C}$ ingresó por la sección baja de la columna de vidrio, mezclándose con el tolueno evaporado y obteniéndose un flujo constante de aire contaminado. El mecanismo de control de este proceso se presentó previamente.

Para validar la concentración de tolueno entre 99 ppm a 200 ppm en la corriente gaseosa, se estudió la influencia de la temperatura y área expuesta del líquido, junto con el caudal del aire de entrada, empleando para ello el método gravimétrico.

\subsubsection{Análisis del efecto del flujo del aire, la temperatura del líquido y su área transversal, en el proceso de evaporación del tolueno}

Los parámetros con influencia en el proceso de evaporación se controlaron bajo lo descrito previamente en la Sección 2.1.1. Para evaluar la influencia de los parámetros, se realizó un diseño experimental, en el cual las variables fueron: temperatura del tolueno, flujo del aire y el espacio transversal de los recipientes con tolueno.

En el recipiente de $2,97 \mathrm{~cm}^{2}$ se introdujeron aproximadamente $40 \mathrm{~g}$ de tolueno. El recipiente se colocó en la columna, se fijó la temperatura de la chaqueta de calentamiento a $18{ }^{\circ} \mathrm{C}$ y el flujo del aire se fijó a 2,5 L/min. Posteriormente, se cerró la columna hasta que transcurran $30 \mathrm{~min}$. Una vez alcanzado el tiempo de espera, se abrió la columna, se retiró el recipiente y se pesó en una balanza analítica. Este proceso se repitió por 3 ocasiones.

El flujo de evaporación del tolueno se determinó considerando la diferencia entre la cantidad evaporada y el valor inicial, dividido para el tiempo de realización de la prueba.

Para comprobar el error generado entre el valor teórico y el experimental, se empleó la Ecuación 1 (Incrópera et al., 2011, p. 453).

$$
n_{A}=A * h *\left(\rho_{A, \text { sat }}-\rho_{A, \text { aire }}\right)
$$

Donde:

$n_{A}$ : Caudal de evaporación, $(\mathrm{kg} / \mathrm{s})$

$h$ : Coeficiente de transferencia de masa, $(\mathrm{m} / \mathrm{s})$

$A$ : Área del recipiente, $\left(\mathrm{m}^{2}\right)$

$\rho_{A, t}$ : Concentración del tolueno saturado, $\left(\mathrm{kg} / \mathrm{m}^{3}\right)$

Con los valores obtenidos del caudal de evaporación del tolueno y de aire contaminado, se empleó el modelo de Caja Fija para la determinación de la concentración del tolueno (De Nevers, 2010, p. 120). La Ecuación 2 describe el modelo de caja fija resultante para los parámetros establecidos:

$$
\frac{q}{u}=c
$$

Donde:

$c$ : Concentración del tolueno en el flujo de salida, $\left(\mathrm{kg} / \mathrm{m}^{3}\right)$

$q$ : Flujo de emisión del contaminante, $\left(\mathrm{kg} / \mathrm{m}^{2 *} \mathrm{~s}\right)$

$u$ : Velocidad del aire, $(\mathrm{m} / \mathrm{s})$

De la aplicación de esta ecuación se obtuvieron 18 valores de concentración con los cuales se analizó si la concentración de tolueno es constante en el tiempo para ambas áreas $\left(12,76 \mathrm{~cm}^{2}\right.$ y $2,97 \mathrm{~cm}^{2}$ ). Posteriormente, se precisó el flujo de evaporación de los recipientes de 2,97 y $12,76 \mathrm{~cm}^{2}$ con una temperatura de la camisa de $25{ }^{\circ} \mathrm{C}$. Por último, se fijó el flujo de aire a 0,5 $\mathrm{L} /$ min y se trabajó con las temperaturas de $18^{\circ} \mathrm{C}$ y $25^{\circ} \mathrm{C}$ y con las áreas mencionadas.

Tras los resultados obtenidos, se seleccionaron los parámetros que permitieron obtener una concentración de tolueno de 99 a 200 ppm, considerando que estos son los valores comunes en la industria de pintura, de acuerdo con Health and Human Service (DHHS, 2015).

Los valores obtenidos en este inciso provienen de un método gravimétrico. Considerando que el método es sujeto de errores, se midió la cantidad de tolueno en la corriente de aire a través del método de carbón activo/cromatografía de gases.

\subsubsection{Determinación de la concentración de tolueno a través de adsorción en carbón activo / cromatografía de gases MTA/MA-030/A92}

El procedimiento de adsorción en carbón activado es un método avalado por el Instituto Nacional de Seguridad e Higiene en el Trabajo (INSHT) para la cuantificación de compuestos orgánicos volátiles. El procedimiento consiste en tomar con un tubo de carbón activo, una cierta cantidad de la corriente contaminada con COVs. Aquí se genera una desorción de la cual se obtiene como producto final el sulfuro de carbono (INSHT, 2005).

La solución obtenida se examinó en un cromatógrafo de gases equipado con un detector de ionización FID. El cromatógrafo empleado fue un Agilent 7890, con una columna HP PONA. 
Los parámetros de análisis se detallan a continuación en la Tabla 1.

Tabla 1. Condiciones del análisis por cromatografía de gases

\begin{tabular}{cllc}
\hline & Parámetro & Magnitud & Unidad \\
\hline \multirow{4}{*}{ Temperatura } & Horno & 150 & $\left({ }^{\circ} \mathrm{C}\right)$ \\
& Detector & 250 & $\left({ }^{\circ} \mathrm{C}\right)$ \\
& Inyector & 230 & $\left({ }^{\circ} \mathrm{C}\right)$ \\
\hline \multirow{3}{*}{ Flujo } & Hidrógeno & 45 & $(\mathrm{~mL} / \mathrm{min})$ \\
& Aire & 450 & $(\mathrm{~mL} / \mathrm{min})$ \\
& Nitrógeno & 5 & $(\mathrm{~mL} / \mathrm{min})$ \\
\hline Volumen & Inyección & 0,5 & $(\mu \mathrm{L})$ \\
\hline
\end{tabular}

Con el fin de cuantificar la eficiencia del método en mención, se analizó la muestra a través de celdas electroquímicas con un Equipo Dräger CMS. Este equipo analiza optoelectrónicamente los productos de la reacción química, y detalla la concentración presente del contaminante (Dräger, 2015, p. 352).

Una vez cuantificada la concentración de tolueno en el aire, se implementó un procedimiento para el tratamiento del aire contaminado.

\subsection{Implementación de un sistema para el tratamiento de corrientes gaseosas con tolueno}

En un contenedor hermético de vidrio de dimensiones: $15 \mathrm{~cm}$ de diámetro y $20 \mathrm{~cm}$ de alto se colocaron dos acoples, uno para la entrada del aire y otro para la salida del aire tratado. Las dimensiones del vidrio fueron escogidas para simular un estudio semejante al presente (Bae et al., 2014, p.32).

\subsection{Valoración del efecto del peróxido de hidrógeno en la eliminación del tolueno}

Al ser el $\mathrm{H}_{2} \mathrm{O}_{2}$ un oxidante débil, se analizó inicialmente el efecto de este compuesto en la corriente contaminada con tolueno. Para lo cual, se inyectó al sistema descrito previamente, un volumen de $20 \mathrm{~mL}$ de este oxidante en una concentración del $30 \%$ (v/v), creando un flujo de $2 \mathrm{~mL} / \mathrm{min}$ y $3 \mathrm{~mL} / \mathrm{min}$ (Bae et al., 2014, p. 32).

Tras una hora en el reactor, se midió el porcentaje de remoción del tolueno de la corriente contaminada. El procedimiento se realizó tres veces.

\subsection{Valoración del efecto del catalizador disulfuro de} hierro II en la eliminación del tolueno

\subsubsection{Caracterización y desarrollo del catalizador}

Para determinar el grado de pureza del disulfuro de hierro II, se sometió al mineral a una difracción de rayos $\mathrm{X}$. El análisis fue llevado a cabo en el equipo D8 Advance Bruker del Laboratorio de Metalurgia Extractiva de la EPN.

A continuación, se trituró, molió y tamizó al disulfuro hasta alcanzar los $150 \mu \mathrm{m}$ de diámetro de partícula. Se colocó el disulfuro tamizado en un vaso de precipitación que contenía alcohol etílico al $98 \%$ y se sometió a ultrasonido por 5 minutos. Posteriormente se lavó con ácido nítrico en concentración 1N (Wang et al., 2002; Bae et al., 2014, p. 31).

\subsubsection{Efecto de la concentración del catalizador disulfuro de hierro II en el tratamiento de la corriente contaminada}

Con los datos obtenidos del efecto del $\mathrm{H}_{2} \mathrm{O}_{2}$ en la corriente contaminada y, una vez escogido el mejor tratamiento, se prosiguió a determinar el efecto del disulfuro de hierro II en el flujo de aire. Para ello, se preparó en $3 \mathrm{~L}$ de agua destilada una suspensión de disulfuro de hierro II, bajo un pH de 2,5. La suspensión se mantuvo en agitación constante durante 24 horas en el sistema descrito previamente. Posteriormente, se inyectó un volumen de $20 \mathrm{~mL}$ de $\mathrm{H}_{2} \mathrm{O}_{2}$ y se encendió la bomba para controlar el caudal de entrada del peróxido (Barriuso y Lévy, 2004, p. 117).

Del estudio de Zhang et al. (2014), se tomaron los valores sugeridos de $10 \mathrm{~g}, 20 \mathrm{~g}$ y $30 \mathrm{~g}$ del catalizador disulfuro de hierro II, para el tratamiento de la corriente de aire. De ello, se realizaron tres mediciones con cada valor del catalizador, y se evaluó la cantidad de tolueno en el aire de salida tras una hora en el reactor, para determinar el porcentaje de remoción (p.1).

Con los valores obtenidos se escogió la concentración de catalizador que generó un mayor porcentaje de eliminación del tolueno en el aire contaminado. Para descartar posibles variaciones en el tiempo, se tomó por triplicado los valores de $\mathrm{pH}$, concentración final de tolueno, y temperatura del sistema cada 2 horas, durante un lapso de 8 horas y otra medida a las 12 horas.

\subsubsection{Estudio de los productos obtenidos en el proceso}

Tras un proceso de oxidación completa, se tiene como productos de la reacción agua $\left(\mathrm{H}_{2} \mathrm{O}\right)$ y anhídrido carbónico $\left(\mathrm{CO}_{2}\right)$. Para verificar la formación de estos compuestos en este estudio, se tomaron tres muestras de $2 \mathrm{~L}$ del aire tratado en un envase impermeable dispuesto de una válvula antiretorno, y se analizaron en un cromatógrafo de gases (Perkin Elmer, Clarus 500). Las dimensiones de la columna fueron $35 \mathrm{~cm}$ de longitud, 0,32 mm de diámetro, modelo 19091H, marca Hp, rellena con material de 0,25 $\mu \mathrm{m}$ (Colin, 2004, p. 505; ASTM D1945-03, 2010).

\section{RESULTADOS Y DISCUSIÓN}

\subsection{Desarrollo de un medio constante de generación de aire contaminado con tolueno}

\subsubsection{Diseño y elaboración del sistema}

a. Control de la temperatura del aire

Inicialmente, se empleó aire de temperatura variable procedente del laboratorio de Análisis Instrumental de la EPN. Con el fin de mantener constante la temperatura, se diseñó y desarrolló un sistema de control de temperatura.

Tomando la temperatura ambiental de la ciudad de Quito, y considerando que la temperatura mínima para el año 2015 fue 
de $9{ }^{\circ} \mathrm{C}$, se escogieron valores de 10 a $19^{\circ} \mathrm{C}$ para la realización de los cálculos y alcanzar los $20^{\circ} \mathrm{C}$. En la Figura 1 se pueden visualizar los datos obtenidos para un caudal igual a $2,5 \mathrm{~L} / \mathrm{min}$.

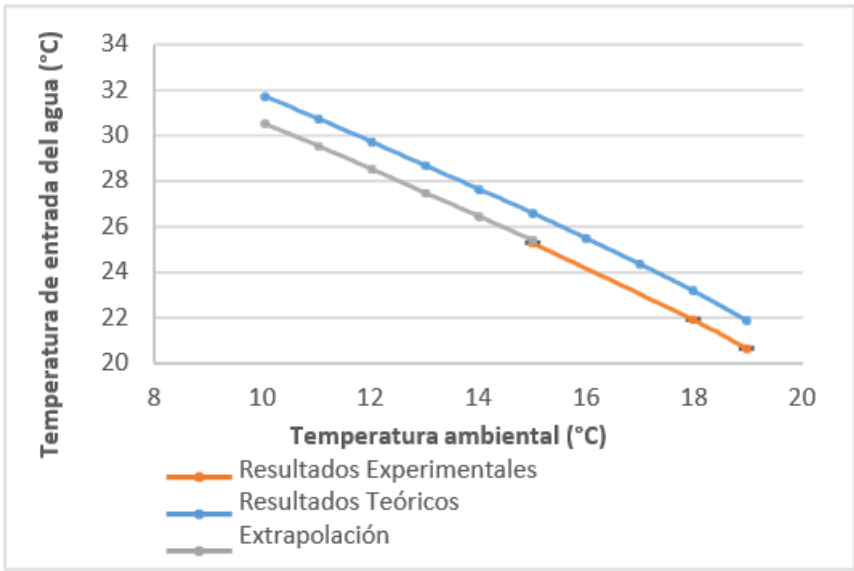

Figura 1. Temperatura ambiental en relación con la temperatura inicial del agua para el intercambiador (Caudal de aire $=2,5 \mathrm{~L} / \mathrm{min}$ )

Del análisis de la Figura 1, se tiene que la máxima temperatura requerida, cuando la temperatura ambiental es $10^{\circ} \mathrm{C}$, es de $31,8{ }^{\circ} \mathrm{C}$. Para alcanzar este valor, se requieren $0,42 \mathrm{~W}$ de energía (Perry, et al., 2001, p. 2).

Dada la variabilidad de la temperatura ambiental, se experimentó con 3 temperaturas: $15^{\circ} \mathrm{C}, 18{ }^{\circ} \mathrm{C}$ y $19{ }^{\circ} \mathrm{C}$. Los valores teóricos están simbolizados por la curva de color azul, mientas que la curva de color naranja muestra los resultados experimentales. Cómo se puede observar, las dos curvas se disponen paralelamente, con una variación aproximada de un grado centígrado entre ambos valores. Esta variación puede considerarse constante, por lo cual se pueden extrapolar los resultados y construir una curva como la que se muestra en color plomo.

Con la curva extrapolada, se pueden obtener datos en caso de variación de la temperatura en el rango de 10 a $15{ }^{\circ} \mathrm{C}$.

b. Control del flujo de aire, temperatura del tolueno, y del área transversal expuesta del líquido

Con el fin de controlar el espacio transversal expuesto del líquido, se tomaron dos envases con una misma altura, pero distinto diámetro. Los valores de su área transversal fueron de $12,76 \mathrm{~cm}^{2}$ y $2,97 \mathrm{~cm}^{2}$. El caudal de aire se controló a través de una válvula de un rotámetro.

Tomando estos parámetros, se diseñó y desarrolló un sistema de una columna de vidrio de medidas $55 \mathrm{~cm}$ de altura y $3 \mathrm{~cm}$ de radio, dentro de la cual se insertaron los envases con tolueno.

En la Figura 2, se puede observar a la columna revestida por una chaqueta de calentamiento, dentro de la cual, circuló agua proveniente de un baño termostático, con la cual se logró mantener constante la temperatura del tolueno.

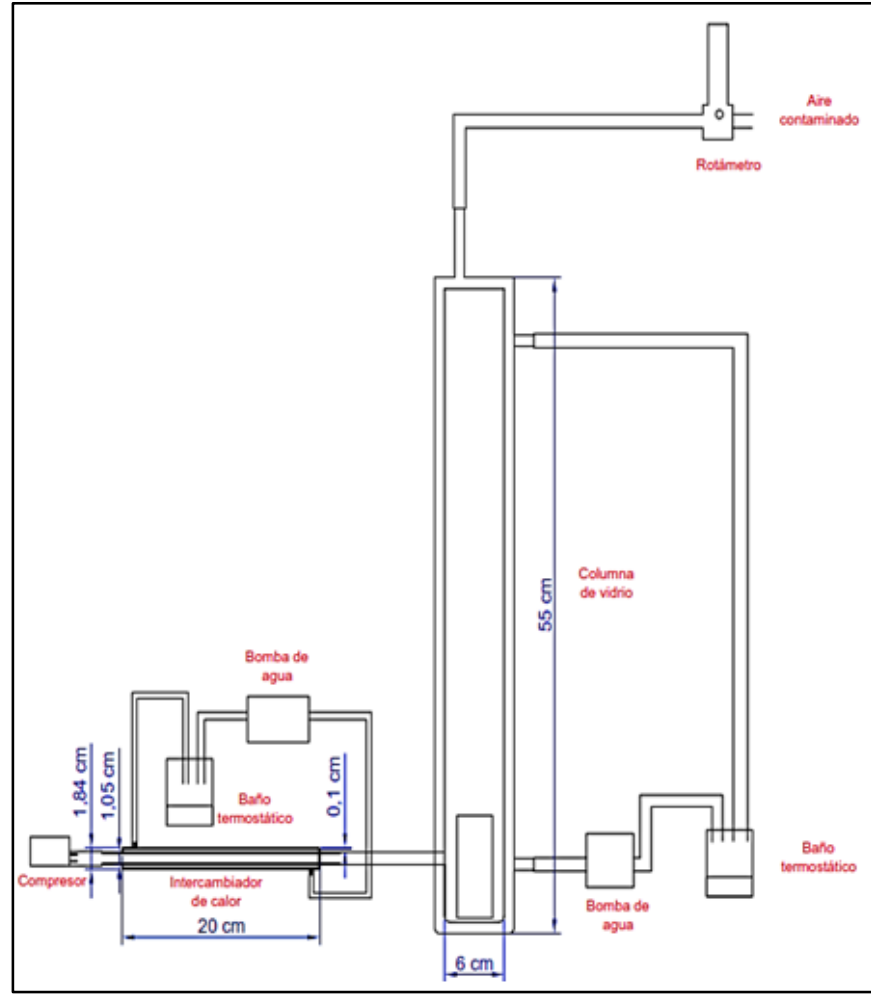

Figura 2. Bosquejo del sistema desarrollado de contaminación de aire con tolueno

3.1.2 Determinación del efecto del flujo de aire, la temperatura del líquido y su área transversal expuesta, en la evaporación del tolueno

En función de los análisis realizados, se determinó que el flujo de evaporación fue de $1,1 \mathrm{mg} / \mathrm{min}$ para un área de $2,97 \mathrm{~cm}^{2}$, con una temperatura del líquido de $18{ }^{\circ} \mathrm{C}$ y $2,5 \mathrm{~L} / \mathrm{min}$ de flujo de aire, mientras que a una temperatura de $25^{\circ} \mathrm{C}$ fue de 3,6 $\mathrm{mg} / \mathrm{min}$. De acuerdo con lo expuesto, se considera que existe una relación directa entre la cantidad de tolueno evaporado y la temperatura del líquido. La evaporación está determinada tanto por la temperatura del líquido como la del aire, sin embargo, en este sistema la temperatura del aire permanece constante mientras que la temperatura del líquido aumenta. El incremento de temperatura genera un cambio en la presión del vapor del líquido, provocando una mayor transferencia de masa (Montoya, 2002, p. 12).

Los resultados obtenidos del análisis de la concentración de tolueno a diferentes áreas transversales y temperaturas se presentan en la Tabla 2. Al comparar los datos obtenidos, se determina una relación directa entre las áreas y la concentración de tolueno. Esto concuerda con lo expuesto por Incrópera et al. (2011), quien menciona que la evaporación aumenta su eficiencia en cuanto mayor es el área expuesta del líquido (p. 453).

Respecto al caudal de evaporación, se obtuvo que a $2,97 \mathrm{~cm}^{2}$; $18{ }^{\circ} \mathrm{C} \mathrm{y} ; 0,5 \mathrm{~L} / \mathrm{min}$ se evaporaron $0,47 \mathrm{mg} / \mathrm{min}$ mientras que a $2,97 \mathrm{~cm}^{2} ; 18{ }^{\circ} \mathrm{C}$ y $2,5 \mathrm{~L} / \mathrm{min}$; se evaporaron $1,11 \mathrm{mg} / \mathrm{min}$. Esto indica una relación directa entre el flujo de aire y la evaporación de tolueno, confirmando la proposición realizada por Pozo (2012, p. 22). 
Tabla 2. Valores de la concentración inicial de tolueno a diferentes temperaturas del líquido, áreas de evaporación y flujos de aire

\begin{tabular}{|c|c|c|c|c|}
\hline & \multicolumn{4}{|c|}{ Temperatura $\left({ }^{\circ} \mathrm{C}\right)$} \\
\hline & & & & \\
\hline $\begin{array}{l}\text { Flujo } \\
\text { (L/min) }\end{array}$ & 2,97 & 12,76 & 2,97 & 12,76 \\
\hline 0,5 & $243 \pm 21$ & $1002 \pm 80$ & $418 \pm 7$ & $1674 \pm 13$ \\
\hline 2,5 & $115 \pm 8$ & $4044 \pm 6$ & $373 \pm 6$ & $8193 \pm 2$ \\
\hline
\end{tabular}

Reconsiderando lo expuesto en el experimental, de estos resultados se eligió el flujo de aire, la temperatura y el área de exposición que permitió una concentración de tolueno de 99 ppm a 200 ppm en la corriente gaseosa. Los resultados obtenidos permitieron una concentración aproximada de 115 ppm a $18{ }^{\circ} \mathrm{C}$ de temperatura; $2,5 \mathrm{~L} / \mathrm{min}$ de aire, y $2,97 \mathrm{~cm}^{2} \mathrm{de}$ área transversal.

Al relacionar los resultados experimentales con los teóricos, se determinó un error del 17,53\%. La diferencia hallada pudo haberse generado por la presunción del flujo paralelo al área de evaporación cuando el mismo es vertical, conforme se presenta en la Figura 2. Por ello, se desarrolló un procedimiento analítico para determinar cuantitativamente la presencia de tolueno en el aire.

\subsubsection{Evaluación del proceso de adsorción de tolueno en carbón activo / cromatografía de gases MTA/MA- 030/A92}

Los resultados de la concentración de tolueno en la corriente de aire a través del procedimiento de adsorción con carbón activo/cromatografía de gases se muestran en la Tabla 3.

Tabla 3. Concentraciones de tolueno en la corriente de aire, obtenidas a través de cromatografía de gases y celdas electroquímicas

\begin{tabular}{cccc}
\hline Pruebas & $\begin{array}{c}\text { Concentración } \\
\text { obtenida por } \\
\text { cromatografía } \\
\text { de gases (ppm) }\end{array}$ & $\begin{array}{c}\text { Concentración } \\
\text { obtenida con } \\
\text { celdas } \\
\text { electroquímicas } \\
(\mathbf{p p m})\end{array}$ & $\begin{array}{c}\text { Error del método } \\
\text { cromatografía de } \\
\text { gases/celdas } \\
\text { electroquímicas } \\
(\%)\end{array}$ \\
\hline 1 & 110 & 119 & 7,3 \\
2 & 105 & 112 & 5,9 \\
3 & 108 & 115 & 5,5 \\
\hline PROM & $108 \pm 2$ & $115 \pm 3$ & $6,2 \pm 0,8$ \\
\hline
\end{tabular}

La concentración determinada a través del método gravimétrico fue $115 \mathrm{ppm}$, mientras que a través de la cromatografía de gases fue $108 \mathrm{ppm}$. La diferencia entre ambos métodos es mínima por lo cual se considera que no hay variación.

De los valores alcanzados con los métodos señalados, se compararon con aquellos obtenidos a través del análisis de celdas electroquímicas con el Equipo Dräger CMS (Dräger, 2015, p. 352). Los resultados que se detallan en la Tabla 3, indican similitudes en los valores alcanzados, con apenas un $6.2 \%$ de diferencia. Esta variación era esperada dado que al desorber el tolueno del carbón activo, una mínima cantidad del mismo se queda adsorbido, como lo menciona la norma MTA/MA-030/A92 (INSHT, 2005). Conforme a la descrito, se acepta el método de carbón activado/cromatografía como confiable y se asume un $93.8 \%$ de confiabilidad en la adsorción y desorción del contaminante, y se utiliza para los análisis posteriores.

\subsection{Desarrollo de un sistema para el tratamiento de aire contaminado con tolueno}

En un sistema conformado por un envase hermético de vidrio de medidas $15 \mathrm{~cm}$ de diámetro y $20 \mathrm{~cm}$ alto, se insertó un acople para la entrada de aire contaminado y otro acople para la salida del aire tratado. Se llenó el recipiente con $3 \mathrm{~L}$ de agua destilada y se acondicionó a un $\mathrm{pH}$ de 2,5 por la adición de ácido sulfúrico $\left(\mathrm{H}_{2} \mathrm{SO}_{4}\right)$ a concentración $1 \mathrm{~N}$. Se añadió el catalizador al inicio del ensayo, y posteriormente por la parte superior del envase de vidrio se insertó $\mathrm{H}_{2} \mathrm{O}_{2}$ a un caudal controlado y finalmente se adaptó un sistema de agitación magnética (Bae, et al., 2014, p. 32).

El caudal de aire con tolueno a una concentración de 108 ppm, ingresó al sistema de tratamiento a 2,5 L/min. El caudal se difundió en el envase, atravesó el medio oxidante y salió por el acople, con un flujo determinado por un rotámetro. La cantidad empleada del oxidante y el catalizador se detalla en las Secciones 2.3 y 2.4 .

\subsection{Evaluación del uso del peróxido de hidrógeno en la remoción del tolueno de la corriente de aire contaminada}

Previo a la utilización del catalizador, se estudió el efecto del peróxido de hidrógeno en el caudal contaminado con tolueno. Para lo cual, se inyectó al sistema, compuesto por un envase de vidrio, peróxido de hidrógeno al $30 \%$ en dos caudales 2 $\mathrm{mL} / \mathrm{min}$ y $3 \mathrm{~mL} / \mathrm{min}$

De los resultados alcanzados se tiene que la degradación del tolueno con peróxido de hidrógeno es baja, con apenas el 10.1 $\%$ al usarse $2 \mathrm{~mL} / \mathrm{min}$ y $10,5 \%$ cuando se empleó un 3 $\mathrm{mL} / \mathrm{min}$.

Considerando que la diferencia de degradación entre los dos flujos es mínima, de apenas el $0,4 \%$, se optó por el caudal de $2 \mathrm{~mL} / \mathrm{min}$.

\subsection{Análisis del uso del catalizador disulfuro de hierro II en el porcentaje de remoción de tolueno de la corriente gaseosa contaminada}

\subsubsection{Caracterización y preparación del catalizador}

Cómo un paso previo al análisis de la influencia del catalizador, se procedió a caracterizar al mineral a través de una difracción de rayos $\mathrm{X}$. De acuerdo a los resultados, el mineral contiene un $86 \%$ de disulfuro de hierro II y un $14 \%$ de minerales como esfalerita, caolinita, phologopita, butlerita; algo típico de hallar en las minas ecuatorianas (Prodeminca, 2000). 


\subsubsection{Análisis de la concentración de disulfuro de hierro II en el tratamiento}

Tomando el caudal de $2 \mathrm{~mL} / \mathrm{min}$ de peróxido de hidrógeno obtenido en pasos previos, se experimentó con los pesos de $10 \mathrm{~g}, 20 \mathrm{~g}$ y $30 \mathrm{~g}$ del catalizador disulfuro de hierro II, considerando como variable al porcentaje de remoción de tolueno. La concentración final de tolueno se midió tras 60 minutos en el reactor. Los valores obtenidos se detallan a continuación en la Tabla 4.

Tabla 4. Porcentajes de degradación y concentración final de tolueno en la emisión contaminada, tras tratamiento con catalizador

\begin{tabular}{ccccc}
\hline $\mathbf{F e S}_{\mathbf{2}} \mathbf{( g )}$ & Prueba & $\begin{array}{c}\text { Concentración } \\
(\mathbf{p p m})\end{array}$ & $\begin{array}{c}\text { Media } \\
(\mathbf{p p m})\end{array}$ & $\begin{array}{c}\text { Degradación } \\
(\boldsymbol{\%})\end{array}$ \\
\hline \multirow{2}{*}{10} & 1 & 71 & & \\
& 2 & 72 & $69 \pm 3$ & 36,25 \\
& 3 & 65 & & \\
20 & 1 & 41 & & 61,09 \\
& 2 & 43 & $42 \pm 1$ & \\
\multirow{3}{*}{30} & 3 & 42 & & 70,39 \\
& 1 & 34 & $32 \pm 1$ & \\
\hline
\end{tabular}

Como se puede evidenciar, el porcentaje de degradación aumenta a mayor cantidad de disulfuro de hierro II, de modo que con $30 \mathrm{~g}$ de $\mathrm{FeS}_{2}$ la remoción de tolueno fue del 70,39\%. Sin embargo, se pueden obtener porcentajes más altos como lo demostró Bae et al. (2014), al alcanzar en su investigación un 90\% de degradación, considerando una concentración inicial de tolueno de 7900 ppm (p. 32).

La diferencia porcentual de degradación ente ambas investigaciones está ligada a la velocidad de reacción, la cual está relacionada con la concentración inicial de reactivos, de modo que, a menor concentración, menor velocidad de reacción (Fogler, 2001, p. 69).

A través del catalizador, se alcanzó una concentración final de tolueno en la emisión contaminada de $32 \mathrm{ppm}$. Con respecto a la temperatura, el sistema alcanzó los $25^{\circ} \mathrm{C}$ con los 10,20 y $30 \mathrm{~g}$ de $\mathrm{FeS}_{2}$, no obstante, el valor del pH varió en función de la cantidad empleada de catalizador de modo que, con $30 \mathrm{~g}$ el valor de $\mathrm{pH}$ descendió de 2,5 a 2,07. Este comportamiento se relaciona con la presencia de pirita en el mineral, cuyo alcance del punto de equilibrio de $\mathrm{pH}$ entre $3-2,5$, produce la liberación de hierro e hidrógeno, mecanismo oxidativo descrito en las Ecuaciones 3,4,5,6 que se presentan enseguida. La presencia de hierro en los reactantes permite llegar al equilibrio de forma más rápida (Che, et al., 2011, p. 1360).

$$
\begin{aligned}
& 2 \mathrm{FeS}_{2}+7 \mathrm{O}_{2}+2 \mathrm{H}_{2} \mathrm{O} \rightarrow 2 \mathrm{Fe}^{2+}+4 \mathrm{SO}_{4}{ }^{2-}+4 \mathrm{H}^{+} \\
& 2 \mathrm{FeS}_{2}+15 \mathrm{H}_{2} \mathrm{O}_{2} \rightarrow 2 \mathrm{Fe}^{3+}+4 \mathrm{SO}_{4}^{2-}+2 \mathrm{H}^{+}+14 \mathrm{H}_{2} \mathrm{O} \\
& \mathrm{Fe}^{2+}+\mathrm{H}_{2} \mathrm{O}_{2} \rightarrow \mathrm{Fe}^{3+}+\mathrm{OH}^{-}+\mathrm{OH} \\
& \mathrm{FeS}_{2}+14 \mathrm{Fe}^{3+}+8 \mathrm{H}_{2} \mathrm{O} \rightarrow 15 \mathrm{Fe}^{2+}+2 \mathrm{SO}_{4}{ }^{2-}+16 \mathrm{H}^{+}
\end{aligned}
$$

Ecuación 4, los cuales al reaccionar forman el catión $\mathrm{Fe}^{3+}$ y liberan hidrógeno y agua. El catión de hierro $\mathrm{Fe}^{2+}$, se combina con el $\mathrm{H}_{2} \mathrm{O}_{2}$ y produce radicales hidroxilos $(\mathrm{OH})$, los cuales, en el paso de buscar el equilibrio, reaccionan con el compuesto orgánico degradándolo (Ecuación 5). Finalmente, el disulfuro de hierro II reacciona con el catión de hierro $\mathrm{Fe}^{3+}$ y como producto de esta reacción se genera nuevamente el catión $\mathrm{Fe}^{2+}$ (Ecuación 6) (Che, et al., 2011, p. 1356).

Con el fin de comprobar la estabilidad del sistema en función del tiempo, se dio seguimiento a los valores de concentración final de tolueno, temperatura y $\mathrm{pH}$, recolectando información cada 2 horas durante 8 horas y una medición final a las 12 horas. Los resultados obtenidos se presentan en las Figura 3, Figura 4 y Figura 5.

En la Figura 3, se visualiza la concentración final de tolueno. Transcurridas las 12 horas la concentración se mantiene constante, concluyendo que el sistema es estable en el tiempo sin perder su eficiencia. El comportamiento descrito es similar al realizado por Bae et al. (2014), en el cual se determinó que un medio para el tratamiento con $\mathrm{FeS}_{2}$ y $\mathrm{H}_{2} \mathrm{O}_{2}$ puede trabajar ininterrumpidamente por 61 días sin perder su eficiencia (p. $31)$.

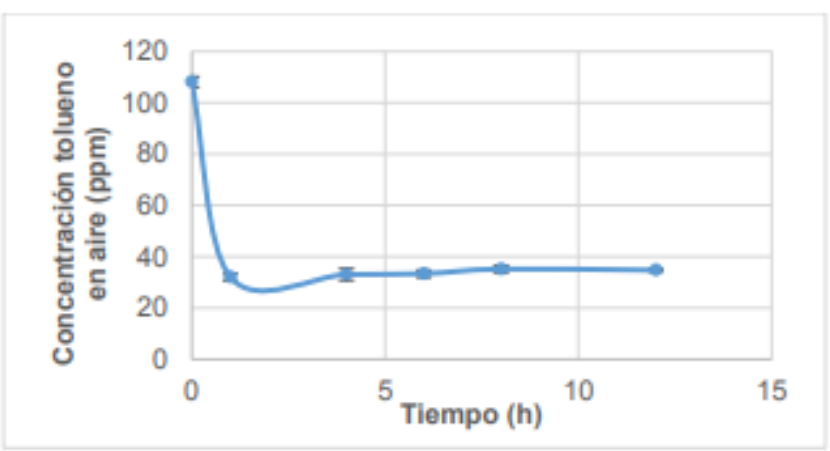

Figura 3. Detalle del comportamiento de la concentración de tolueno vs tiempo $\left(\mathrm{FeS}_{2}=30 \mathrm{~g}\right)$

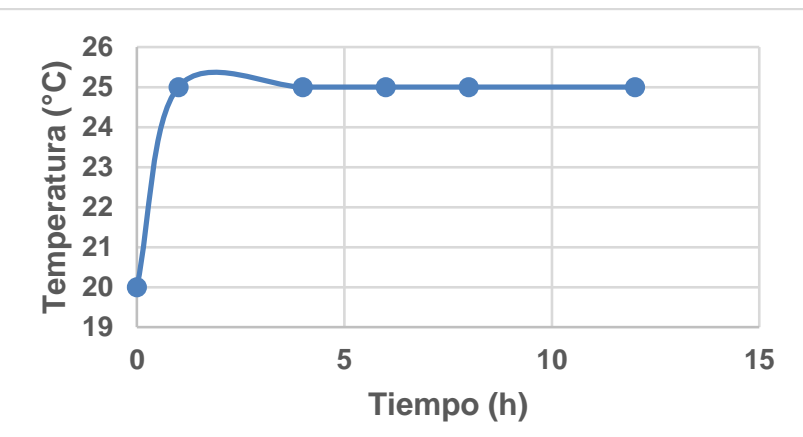

Figura 4. Temperatura sistemática vs tiempo $\left(\mathrm{FeS}_{2}=30 \mathrm{~g}\right)$

En la Ecuación 3 se muestra la reacción entre el disulfuro de hierro II (sólido), el oxígeno en estado gaseoso y el agua, cuyos productos de la reacción tiene, entre otros, al catión de hierro $\left(\mathrm{Fe}^{2+}\right)$. La reacción entre el $\mathrm{FeS}_{2}$ y el $\mathrm{H}_{2} \mathrm{O}_{2}$ se presenta en la 


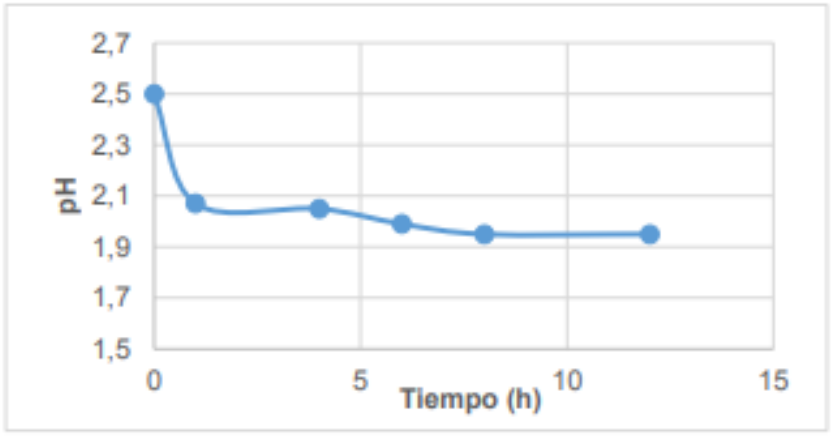

Figura 5. $\mathrm{pH}$ del medio vs tiempo $\left(\mathrm{FeS}_{2}=30 \mathrm{~g}\right)$

En la Figura 4 se puede observar que la temperatura permanece constante a $25^{\circ} \mathrm{C}$. El incremento en la primera hora se genera por la necesidad de la reacción de alcanzar el punto de equilibrio en cuanto a la formación de iones hidroxilos (Bae et al., 2014, p. 32).

En la Figura 5 se visualiza el comportamiento del pH en función del tiempo. Como se puede observar en la primera hora existe una caída en su valor hasta alcanzar el equilibrio, valor a partir del cual no existen variaciones sustanciales. Este resultado es similar al conseguido por Bae et al. (2014). Dada la estabilidad del sistema, el mismo resulta efectivo, pues si el $\mathrm{pH}$ aumentara por la presencia de radicales hidroxilos, se perdería completamente la eficiencia debido a la precipitación de los iones disueltos formando óxidos de hierro (Bae et al., 2014,p. 34).

\subsubsection{Análisis de los productos del proceso oxidativo}

Los productos de una oxidación completa son el agua y el dióxido de carbono. Para confirmar la formación de estos compuestos a partir del tolueno, se tomó por triplicado muestras del aire de salida tratado y se analizaron conforme la norma ASTM D1945-03 (ASTM, 2010).

Es menester la determinación de los componentes en el aire tratado, pues los mismos van en función del contaminante, de modo que si el contaminante está compuesto exclusivamente por moléculas de hidrógeno y carbono se obtendrán como productos agua y dióxido de carbono, de lo contrario, se formarán otros compuestos cómo óxidos de azufre, nitrógeno, o vapores ácidos (EPA, 2002, p. 2).

De los análisis se obtuvo que el componente en mayor porcentaje fue el nitrógeno en un 98,21\%, procedente del aire que ingresa al sistema de evaporación. La concentración del agua alcanzó un 1,57 \%, considerado un valor bajo; su presencia se puede justificar por la humidificación de la corriente gaseosa al atravesar el lecho oxidante, o como resultado de la oxidación. En cuanto al porcentaje de dióxido de carbono, se encontró un $0,21 \%$, generado por el proceso de oxidación del tolueno (Colin, 2004, p. 505).

Existe una gran dificultad en cuantificar los valores de dióxido de carbono y agua formados por la oxidación, debido a que la columna empleada para detectar la presencia de nitrógeno y oxígeno arroja un solo pico.
Otras investigaciones similares de tratamientos con disulfuro de hierro II, han obtenido porcentajes del 38,8\% de presencia del dióxido de carbono en los productos. Esto podría suponer que en la presente investigación algunos compuestos no alcanzaron la oxidación completa (Bae, et al., 2014).

Considerando los resultados alcanzados en este trabajo, se considera que la acción de catálisis con disulfuro de hierro II, representa una alternativa viable y eficiente para gestionar las emisiones gaseosas con tolueno procedentes de industrias de pintura, evitando que la población que mantiene contacto con este contaminante vea afectada su salud (INSHT, 2007, p. 2; IDEAM, 2003).

\section{CONCLUSIONES}

Se alcanzó una concentración final de tolueno de 32,02 ppm, como resultado de una degradación del 70,39\% del valor inicial, con el empleo de 30 gamos del catalizador de disulfuro de hierro II, con lo cual se disminuye el riesgo asociado a la salud, generado por la exposición a este contaminante.

Existe una relación directa entre la temperatura del líquido y la evaporación del tolueno, cuando se mantiene constante el flujo de aire y el área transversal, de modo que la cantidad evaporada fue de $1,1 \mathrm{mg} / \mathrm{min}$ a $18{ }^{\circ} \mathrm{C}$ mientras que, a $25^{\circ} \mathrm{C}$, el caudal de evaporación fue de 3,6 $\mathrm{mg} / \mathrm{min}$.

Al mantener constante la temperatura y el área transversal, se deriva una relación inversa entre el flujo de aire y la concentración final de tolueno, a manera que a $2,5 \mathrm{~L} / \mathrm{min}$ se obtiene una concentración de $115 \mathrm{ppm}$ de tolueno, y a 0,5 L/min, 373 ppm.

La concentración de tolueno en el flujo de aire determinado a través del método gravimétrico a condiciones de temperatura de $18{ }^{\circ} \mathrm{C}, 0,5 \mathrm{~L} / \mathrm{min}$ de caudal de aire y $12,76 \mathrm{~cm}^{2}$ de área, fue de $1002 \mathrm{ppm}$, mientras que con un área de 2,97 cm2 se determinó una concentración de 243 ppm. De acuerdo con lo expuesto se determina una relación directa entre la concentración y el área transversal.

El peróxido de hidrógeno por sí solo no es un método eficiente de degradación de tolueno, pues tras la experimentación, al tratar una corriente de $2 \mathrm{~mL} / \mathrm{min}$ el porcentaje degradado fue del $10,1 \%$, mientras que para un flujo de $3 \mathrm{~mL} / \mathrm{min}$ fue $10,5 \%$.

A través de una difracción de rayos $\mathrm{X}$, se determinó en el mineral un $86 \%$ de disulfuro de hierro II y un $14 \%$ de esfalerita, caolinita, butlerita y phologopita.

Existe una relación inversa entre la concentración del catalizador y la cantidad final del contaminante, de modo que al emplear $10 \mathrm{~g}, 20 \mathrm{~g}$ y $30 \mathrm{~g}$ de disulfuro de hierro II, la concentración fue disminuyendo de 69 ppm, a 42 ppm y 32 ppm.

El tratamiento con 30 gramos de $\mathrm{FeS}_{2}$ en la corriente contaminada con tolueno, genera un sistema estable en el tiempo, manteniendo constantes los parámetros de temperatura y $\mathrm{pH}$, a $25^{\circ} \mathrm{C}$ y 2,07 respectivamente. 
Se obtuvieron como productos finales del tratamiento de la corriente contaminada, al dióxido de carbono y agua, como resultado de una oxidación.

\section{REFERENCIAS}

Acedo, J. (2006). Instrumentación y control avanzado de procesos. Teoría y Práctica (1). Madrid, España: Día Santos.

ASTM. (2010). Standard Test Method for Analysis of Natural Gas by Gas Chromatography. (marzo, 2016).

Bae, C., Choi, K., y Lee, W. (2014). Degradation of off-gas toluene in continuous pyrite Fenton system. Journal of Hazardous Materials, 280 (1), 31-37.doi: 10.1016/j.jhazmat.2014.07.054

Barriuso, F., y Lévy, E. (2004). Diccionario de física (Ed. Económica). (1ra ed.). España: Akal.

Cárdenas, B., Revah, S., Hernández, S., Martínez, A., y Gutiérrez, V. (2003) Tratamiento biológico de compuestos orgánicos volátiles de fuentes fijas. (1ra. ed.). México: Cenica.

Castells, X. (2012). Reciclaje de Residuos Industriales: Residuos sólidos urbanos y fango de depuradora. (2da. Ed.)España: Díaz Santos.

Che, H., Bae, S., y Lee, W. (2011). Degradation of trichloroethylene by Fenton reaction in pyrite suspension. Journal of Hazardous Materials, 185(2-5) 1355-1361. doi: 10.1016/j.jhazmat.2010.10.055

Cobo, J. (2013). Caracterización de la exposición a solventes en los preparadores de pintura en una comercializadora de pinturas (Tesis de Maestría no publicada). Universidad Tecnológica Equinoccial. Quito, Ecuador.

Colin, B. (2004). Environmental Chemistry. (4ta ed.). New York: University Ontario.

De Nevers, N. (2010). Air pollution control engineering. (2da ed.). Illinois, Estados Unidos de Norte América: Waveland press.

Departamento de Salud y Servicios Humanos DHHS. (2015). Draft Toxicological Profile for Toluene. Recuperado de: http://www.atsdr.cdc.gov/toxprofiles/tp56.pdf (mayo, 2019)

Dräger. (2015). Manual de Tubos Dräger/CMS. Recuperado de:http://www.afalpi.com/images/Fichas\%20Tecanicas/Deteccion \%20de\%20Gases Tubos\%20Drager20Manual.pdf (marzo,2019)

EPA. (2000). Technical Overview of Volatile Organic Compounds. Recuperado de: https://www.epa.gov/indoor-air-qualityiaq/technical-overview-volatile-organic-com pounds\#8 (marzo,2019).

EPA.

(2002).

Incineradores.

Recuperado de:https://www3.epa.gov/ttn/catc/dir2/cs3-2ch2-s.pdf (junio, 2019).

EPA. (2004). Condesadores Refrigerados para el Tratamiento de Emisiones Gaseosas. Recuperado

https://www3.epa.gov/ttn/catc/dir2/frefrigs.pdf (junio, 2019)

EPA. (2012). TOLUENE. Recuperado de https://www3.epa.gov/ttn/atw/hlthef/tolue ne.html (junio,2019)

Fogler, S. (2001). Elementos de Ingeniería de las Reacciones Químicas. (3ra ed.). México: Pearson educación.

IDEAM. (2003). Guías para Manejo Seguro y Gestión Ambiental de 25 Sustancias Químicas Peligrosas y Guías Ambientales de Almacenamiento y Transporte por Carretera de Sustancias Químicas Peligrosas y Residuos Peligrosos. Bogota, Colombia: El Ministerio.
IFA. (2007). Valoración del Impacto en Salud en el DMQ. Recuperado de: https://www.hitpages.com/doc/4511783318454272/3\#pageTop (marzo, 2019).

INAMHI (2018). Boletín Climatológico Anual 2018. Instituto Nacional de Meteorología e Hidrología, Quito, Ecuador.

Incrópera, F., DeWitt, D., Bergman, T., y Lavine, A. (2011). Fundamentals of Heat and Mass Transfer. (7ma ed.). Jefferson City, Estados Unidos de América: John Wiley \& Sons.

INSHT (2005). Determinación de hidrocarburos aromáticos (benceno, tolueno, etilbenceno, p-xileno, 1,2,4-trimetilbenceno) en aire Método de adsorción en carbón activo / Cromatografía de gases. Recuperado de: http://www.insht.es/InshtWeb/Contenidos/Documentacion/Fichas Tecnicas/Metodos Analisis/Ficheros/MA/MA_030_A92 .pdf (marzo, 2019).

INSHT (2007). Tolueno: Documentación Toxicológica para el Establecimiento del Límite de Exposición Profesional del Tolueno. Recuperado de http://www.insht.es/InshtWeb/Contenidos/Documentacion/Textos Online/Valores_L imite/Doc_Toxicologica/FicherosSerie2/DLEP\%2039.pdf (junio, 2019).

Kern, D. (1999). Procesos de Transferencia de Calor. (1ra ed.) México: CECSA.

Klein, C., y Hurbut, C. (2002). Manual de mineralogía (4ta. ed.). Madrid: Reverté.

Ministerio del Ambiente (2015). Texto Unificado de Legislación Secundaria Del Ministerio del Ambiente. Libro VI de la Calidad Ambiental, Anexo IV:Norma de Calidad del Aire Ambiente. Ecuador.

Montoya, J. (2002). Efectos ambientales y socioeconómicos por el derrame de petróleo en el río Desaguadero (1ra. ed.). La Paz. Bolivia: Pieb

Perry, R., y Green, D. (2001). Manual del Ingeniero Químico. Madrid, España: Mc Graw Hill.

Pozo, C. (2012). Pérdidas por Evaporación de Xileno y Tolueno (Proyecto de titulación no publicado). Universidad Central, Quito, Ecuador.

Prodeminca. (2000). Evaluación de Distritos Mineros del Ecuador. Ministerio de Energía y Minas. Subsecretaría de Minas.

Wang, k., Jehng, J., Hsieh, Y., y Chang, C. (2002). The reaction pathway for the heterogeneous photocatalysis of trichloroethylene in gas phase. Journal of hazardous materials, 90(1), 63-75. doi: 10.1016/S03043894(01)00331-4.

Zhang, Y., Dai, C., Zhou, X., y Si, H. (2014). An enhanced Fenton reaction catalyzed by natural heterogeneous pyrite for nitrobenzene degradation in an aqueous solution. Chemical Engineering Journal. 244(1), 438-445. doi: 10.1016/j.cej.2014.01.088 


\section{BIOGRAFÍAS}

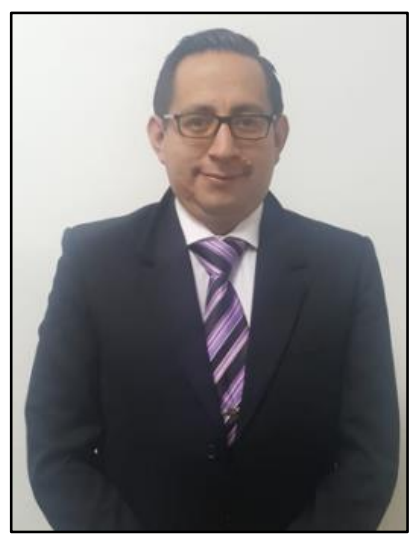

Marcelo F. Cabrera J., nacido el 05 de marzo de 1986 en Quito-Ecuador, estudios completos de pregrado en la Escuela Politécnica Nacional, obteniendo el título de Ingeniero Químico Aprobado Cum Laude. Título de posgrado en la Universidad Internacional SEK como Magíster en Gestión Ambiental. Ha trabajado en Incinerox, Chemeng, Unidad Educativa Jean Jacques Rousseau, Escuela Politécnica Nacional y Universidad Internacional del Ecuador donde hasta la actualidad labora como docente en la Escuela de Gestión Ambiental en la UIDE y Coordinador del Laboratorio de Análisis Instrumental de la EPN. Ha realizado varias publicaciones en revistas LatinIndex y realizado varias conferencias sobre contaminación ambiental y cursos de perfeccionamiento docente.

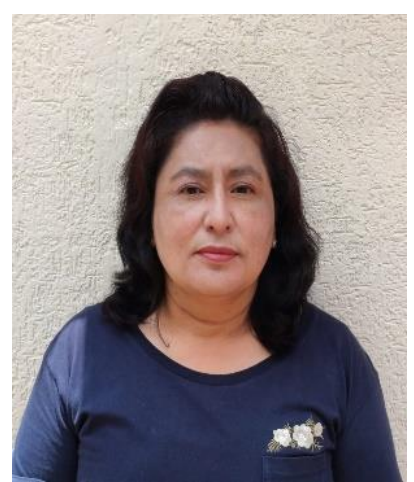

\section{Lucía Margarita}

Montenegro Aguas, Ingeniera

Química, Magister en Ingeniería Ambiental ha sido docente por 24 años en la EPN de las cátedras de Transferencia de Calor, Control de la Contaminación del Aire, entre otras. Es miembro de Green Woman Internacional y cuenta con la Certificación de Evaluador de la Norma ISO 17025 para laboratorios. En referencia a las publicaciones las ha realizado en la "Revista Politécnica" y en la "1st International Conference on Water and Sustainability" con temas relacionados al Tratamiento de aguas y Remediación de Suelos. Ha dictado más de 20 seminarios y conferencias de Monitoreo Ambiental; y dirigido más de 40 proyectos de titulación en temas de Transferencia de Calor, Refinación del Petróleo y Contaminación Ambiental.

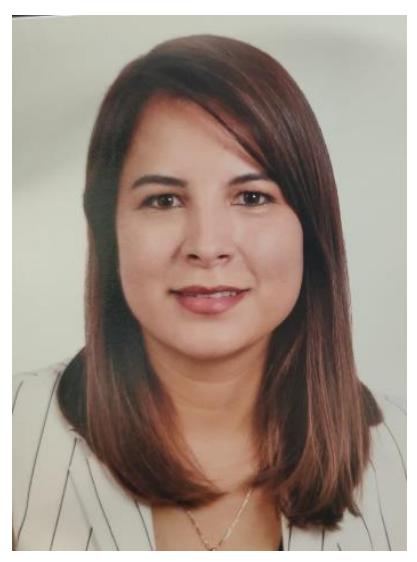

Stephanie Salomé Mejía, Ingeniera de Química especializada en Petróleos y Polímeros graduada en el 2017 de la Escuela Politécnica Nacional Quito-Ecuador. Inicia su carrera en la Industria Petrolera como Ingeniera de Reservorios en el proyecto Samarang, Malasia-Kuala Lumpur Schlumberger-SPM. Trabajó en el área de Subsuelos enfocada en la simulación de Reservorios en proyectos de recuperación primaria y EOR. En el 2018 fue asignada al proyecto Shaya Ecuador-Quito al grupo de Subsuelo Ingeniería de Reservorios, a cargo de la simulación de Reservorios con proyectos de recuperación secundaria. En el 2019 fue asignada al grupo de desarrollo como Ingeniería de Reservorios, a cargo del seguimiento y evaluación de proyectos de recuperación secundaria, identificación de oportunidades W.O, pozos nuevo y optimización producción. Posee 4 publicaciones internas en SLB. 